\title{
Consumo de alimentos ultraprocessados e fatores associados em adultos: evidências do Inquérito ISACamp 2008-2009
}

\author{
Consumption of ultra-processed foods and associated factors \\ in adults: evidence from the 2008-2009 Campinas Health Survey
}

\begin{abstract}
Mayara Gonçalves Pereira (https://orcid.org/0000-0001-7234-1398) ${ }^{1}$
Daniela de Assumpção (https://orcid.org/0000-0003-1813-996X) ${ }^{2}$

Marilisa Berti de Azevedo Barros (https://orcid.org/0000-0003-3974-195X) ${ }^{2}$

Lia Thieme Oikawa Zangirolani (https://orcid.org/0000-0002-9966-5701) ${ }^{3}$
\end{abstract}

${ }^{1}$ Curso de Nutrição, Universidade Federal de São Paulo (UNIFESP). R. Silva Jardim 136, Vila Mathias. 11015-020 Santos SP Brasil. mayaragpereira5@ gmail.com

${ }^{2}$ Centro Colaborador em Análise de Situação de Saúde, Departamento de Saúde Coletiva, Faculdade de Ciências Médicas, Universidade Estadual de Campinas. Campinas SP Brasil.

${ }^{3}$ Laboratório de Segurança Alimentar e Nutricional e Políticas Públicas, Departamento de Políticas Públicas e Saúde Coletiva, UNIFESP. Santos SP Brasil.

\begin{abstract}
The aim of the present study was to evaluate the consumption of ultra-processed (UP) foods and associated factors among adults. We used cross-sectional data on 947 adults from the 2008-2009 Campinas Health Survey. Food consumption data were collected using the 24-h dietary recall method and food items were classified according to NOVA classification based on the nature, extent and purpose of industrial processing. Linear regression models were run to evaluate the association between the consumption UP foods and predictor variables with a $5 \%$ significance level. The average daily energy intake per capita was $2000.6 \mathrm{kcal}$ and UP foods represented $24.1 \%$ of this intake. UP food consumption was higher among women and increased with the increase in schooling. Consumption was also higher among young adults between 20 and 29 years old as well as ex-smokers and individuals who were physically active at leisure. The results show that there is still time to intervene in favor of the health of the adult population. Thus, studies dedicated to the investigation of food intake from the perspective of the NOVA classification, the possible repercussions for health and the evaluation of food and nutrition actions and policies should be prioritized in the current context of Brazil.
\end{abstract}

Key words Nutritional epidemiology, Health surveys, Food consumption, Industrialized foods, Ultra-processed foods
Resumo O objetivo do estudo foi avaliar o consumo de alimentos ultraprocessados (UP) e fatores associados em adultos. Trata-se de um estudo transversal de base populacional, com dados do Inquérito de Saúde de Campinas (ISACamp) 2008-2009. Analisou-se dados de 947 adultos, avaliou-se o consumo alimentar por recordatório de $24 \mathrm{~h}$ e classificou-se os alimentos utilizando a NOVA classificação baseada na natureza, extensão e propósito do processamento industrial de alimentos. O teste de associação entre o consumo de UP e as variáveis preditoras utilizou modelo de regressão linear múltiplo com significância à 5\%. O consumo energético médio foi 2.002,6 kcal per capita/dia, das quais $24,1 \%$ atribui-se aos UP. O consumo de UP foi maior em mulheres e aumentou conforme o grau de escolaridade, também foi maior entre os adultos jovens, entre 20 e 29 anos, assim como entre os ex-fumantes e os fisicamente ativos no lazer. Os resultados revelam que ainda há tempo de intervir em favor da saúde da população adulta. Nesse sentido, pesquisas que se dediquem a estudar o consumo alimentar na perspectiva da NOVA classificação de alimentos, as possiveis repercussões para saúde e a avaliação das ações e politicas de alimentação e nutrição devem ser prioritárias no contexto atual do país.

Palavras-chave Epidemiologia nutricional, Inquéritos de saúde, Consumo de alimentos, Alimentos industrializados, Alimentos ultraprocessados 


\section{Introdução}

A ciência da nutrição construiu-se historicamente centrada no aspecto biológico, analisando os efeitos dos nutrientes sobre a saúde em decorrência das graves carências nutricionais presentes em diversos países até o século $X^{1}$. No cenário científico contemporâneo, a ciência da nutrição se revê e amplia seu ponto de vista, considerando outras dimensões importantes da alimentação como as sociais, culturais, econômicas, ambientais e mais recentemente a tecnológica, com o avanço do processamento dos alimentos ${ }^{2}$.

Com o advento da industrialização, especialmente na segunda metade do século XX, o processamento de alimentos desenvolveu-se rapidamente diante das novas descobertas da ciência e da tecnologia. A natureza e a extensão do processamento industrial de alimentos modificaram-se com o tempo e, nos dias atuais, quase todos os alimentos e bebidas são processados de alguma forma. No entanto, as diferenças entre a natureza, extensão e o propósito do processamento são relevantes para a saúde humana ${ }^{1,3}$.

Diante do cenário global contemporâneo de profundas mudanças no hábito alimentar da população, concomitante ao crescimento da obesidade e outras doenças crônicas não transmissíveis (DCNT), emergiu a necessidade de uma nova diretriz alimentar. Nesse sentido Monteiro et al. ${ }^{4}$ propuseram classificar os alimentos em quatro grupos de acordo com a natureza, extensão e o propósito do processamento industrial.

O primeiro grupo é constituído pelos alimentos in natura ou minimamente processados, que são submetidos a processos que não alteram a composição nutricional, como limpeza, congelamento, pasteurização, entre outros. O segundo grupo é representado pelos alimentos processados, que são alimentos in natura adicionados de sal, açúcar e eventualmente óleo e/ou vinagre. Ingredientes culinários como açúcar, sal, óleo e manteiga representam o terceiro grupo, e devem ser utilizados com moderação nas preparações culinárias. O grupo dos ultraprocessados é composto por formulações que contém pouco ou nenhum alimento in natura, acrescidas de sal, gorduras, açúcares, conservantes e aditivos. São produtos alimentícios prontos para comer ou aquecer, altamente palatáveis e de baixa densidade nutricional ${ }^{4}$.

A literatura aponta que o aumento do consumo de produtos ultraprocessados contribui significativamente para a pandemia de excesso de peso e a ascensão de outras DCNT associadas a uma alimentação de má qualidade ${ }^{1,3-7}$. De acordo com Martins et al. ${ }^{8}$, a participação de produtos ultraprocessados no total de calorias adquiridas nos domicílios brasileiros vem aumentando no decorrer dos anos, passando de 20,8\% em 20022003 para 25,4\% em 2008-2009. Em 19 países da Europa, entre 1991 e 2008, a disponibilidade domiciliar de produtos ultraprocessados variou de $10,2 \%$ em Portugal a 50,4\% no Reino Unido 9 , no México o Inquérito Nacional de Saúde e Nutrição de 2012, apontou $29,8 \%{ }^{10}$, e no Canadá $47,7 \%$ em $2004^{11}$. Na França, uma coorte conduzida de 2009 a 2017, com adultos, mostrou um consumo de ultraprocessados de 35,9\% em relação ao consumo total de energia, e que o aumento no consumo desses alimentos associou-se à um risco de mortalidade global maior, na população de adultos com mais de 45 anos $^{12,13}$.

Diante do crescimento do consumo de produtos alimentícios ultraprocessados e da associação com maior a incidência de obesidade e DCNT ${ }^{4}$, torna-se necessário identificar os segmentos da população mais vulneráveis ao consumo destes produtos. Portanto, o objetivo deste estudo foi avaliar o consumo de alimentos ultraprocessados de acordo com variáveis sociodemográficas, de comportamentos relacionados à saúde e Índice de Massa Corporal, em adultos de 20 a 59 anos.

\section{Métodos}

Foram analisados os dados do Inquérito de Saúde do Município de Campinas, São Paulo (ISACamp), realizado entre fevereiro de 2008 e abril de 2009. Trata-se de um estudo transversal de base populacional que teve como objetivo avaliar as condições de vida e de saúde de três subgrupos da população: adolescentes (10 a 19 anos), adultos (20 a 59 anos) e idosos (60 anos ou mais). Para este estudo, optou-se por analisar os dados referentes ao estrato de adultos.

A amostra foi obtida por procedimentos de amostragem probabilística, estratificada, por conglomerados e em dois estágios: setor censitário e domicílio. No primeiro estágio, procedeu-se o sorteio de 50 setores censitários com probabilidade proporcional ao tamanho, dado pelo número de domicílios. No segundo estágio foi realizado o sorteio sistemático dos domicílios.

O número de pessoas para compor a amostra foi definido considerando à máxima variabilidade para a frequência dos eventos estudados $(\mathrm{p}=0,50)$, nível de $95 \%$ de confiança na determinação dos intervalos de confiança $(z=1,96)$, erro de amostragem entre $4 \%$ e $5 \%$ e efeito de delineamento de 2, resultando em 1.000 indivíduos. Es- 
perando $80 \%$ de taxa de resposta, o tamanho da amostra foi corrigido para 1.250. Para alcançar esse tamanho amostral foram selecionados, de forma independente, 700 domicílios para adultos. As entrevistas foram realizadas com todos os moradores que tinham entre 20 e 59 anos, em cada um dos domicílios selecionados.

Entrevistadores treinados coletaram as informações por meio de um questionário estruturado em 14 blocos temáticos, testado em estudo piloto. Foram levantadas informações demográficas, socioeconômicas, de estilo de vida, uso de serviços de saúde, morbidades, entre outras. Os dados de consumo alimentar foram obtidos por meio de um Recordatório de $24 \mathrm{~h}$ - R24h, utilizando-se o método passo a passo, adaptado de Thompson e Byers $^{14}$. No passo 1, os entrevistadores anotavam os horários e os alimentos/bebidas ingeridos, sem interromper o indivíduo; no passo 2 , ao término do relato espontâneo, o entrevistador perguntava "há algo mais que o sr.(a) se lembre?"; no passo 3 era registrado o nome da refeição; em seguida, no passo 4 os entrevistadores coletavam informações sobre as características dos alimentos/preparações culinárias e, posteriormente, registravam as quantidades ingeridas, em unidades ou medidas caseiras. Por fim, no passo 5 realizava-se a revisão do R24h com o entrevistado. Para minimizar e corrigir possíveis falhas no preenchimento do recordatório, uma nutricionista acompanhou o trabalho de campo. Os alimentos/preparações registrados em unidades ou medidas caseiras foram transformados em gramas ou mililitros, com auxílio de tabelas de medidas caseiras ${ }^{15,16}$.

Os dados dos R24h foram inseridos no programa Nutrition Data System for Research (NDS -R), versão 2007 (NCC - Nutrition Coordinating Center, Universidade de Minnesota, EUA), que utiliza a tabela USDA National Nutrient Database for Standard Reference como fonte de informações para estimar a composição dos alimentos. A opção por este software se deu em virtude do mesmo conter uma vasta opção de alimentos (mais de 18.000) e marcas de produtos (7.000), ademais permite gerar bancos de dados específicos que propiciam o desenvolvimento de análises com foco no consumo tanto de alimentos, como de nutrientes e refeições, por indivíduo. Para além disso, o NDS-R possibilita ao usuário adicionar preparações culinárias em um arquivo de receitas (User-recipe), armazenado separadamente da sua base de dados, sendo possível incluir alimentos/preparações próprias de cada cultura.

Os alimentos referidos no R24h foram organizados de acordo com a classificação de alimentos $\mathrm{NOVA}^{4}$, em quatro grupos: in natura ou mi- nimamente processados, ingredientes culinários, processados e ultraprocessados. Todos os 457 alimentos mencionados foram codificados em uma planilha Excel ${ }^{\circledR}$, por exemplo: o número 11 foi atribuído para frutas, 12 para hortaliças, 13 para leite, 14 para arroz, 15 para carnes vermelhas, e assim por diante; posteriormente, para as análises, esses números foram reunidos em um único código que representasse o grupo de alimentos in natura ou minimamente processados. A organização dos alimentos em cada grupo apoiou-se na proposta de estudos prévios ${ }^{8,17}$, visando a comparabilidade dos resultados.

As variáveis independentes utilizadas para a análise dos fatores associados ao consumo de produtos alimentícios ultraprocessados foram: Sociodemográficas: sexo, faixa etária (em anos), situação conjugal, número de filhos, escolaridade (em anos de estudo), renda familiar per capita (em salários mínimos) e posse de plano de saúde.

Comportamentos relacionados à saúde: frequência de ingestão de bebidas alcoólicas, tabagismo e prática de atividade física em contexto de lazer. Os indivíduos foram categorizados em ativos (aqueles que praticam ao menos $150 \mathrm{mi}$ nutos por semana, distribuídos, no mínimo, por três dias), insuficientemente ativos (os que praticavam menos de 150 minutos por semana, ou praticam mais, porém em menos de três dias na semana) e inativos (os que não realizavam qualquer exercício físico).

Índice de Massa Corporal (IMC): calculado com informações referidas de peso e altura, e classificado em baixo peso (IMC $<18,5 \mathrm{~kg} / \mathrm{m}^{2}$ ), eutrofia (IMC $\geq 18,5 \mathrm{e}<25 \mathrm{~kg} / \mathrm{m}^{2}$ ), sobrepeso (IMC $\geq 25$ e $<30 \mathrm{~kg} / \mathrm{m}^{2}$ ) e obesidade $\left(\text { IMC } \geq 30 \mathrm{~kg} / \mathrm{m}^{2}\right)^{18}$.

Para as análises estatísticas, inicialmente foram calculadas as médias de contribuição energética de cada grupo alimentar e dos respectivos alimentos em relação ao valor energético total. Em seguida, estimou-se as médias de consumo e frequência relativa da contribuição energética dos alimentos ultraprocessados segundo as categorias das variáveis independentes. A associação com as variáveis independentes foi verificada por meio de regressão linear, com intervalo de confiança de 95\%. Foi desenvolvido um modelo de regressão linear múltipla, no qual foram inseridas as variáveis com alguma categoria apresentando nível de significância inferior a 0,20 na análise bivariada, tendo permanecido no modelo aquelas com $\mathrm{p}<0,05$.

As análises foram feitas no módulo Survey (svy) do software Stata 12.0. O projeto ISACamp 2008/2009 foi aprovado pelo Comitê de Ética em Pesquisa da Faculdade de Ciências Médicas da 
Universidade Estadual de Campinas, e todos os entrevistados assinaram o Termo de Consentimento Livre e Esclarecido.

\section{Resultados}

Foram entrevistados 949 adultos de 20 a 59 anos de idade, sendo $53,2 \%$ mulheres. A média de idade da população foi de 37,5 anos (IC95\%: 36,6$38,3)$ e a média de consumo energético diário foi de 2002,6 kcal (IC95\%: 1923,9-2081,2) para o conjunto dos adultos, sendo 2266,8 kcal (IC95\%: $2149,8-2383,7)$ para os homens e $1753,6 \mathrm{kcal}$ (IC95\%: 1670,2-1837,1) para as mulheres.

$\mathrm{Na}$ Tabela 1 observa-se que os alimentos in natura contribuíram com $54,1 \%$ da ingestão energética total, e os ultraprocessados com $24,1 \%$. Dentre os alimentos in natura ou minimamente processados destacam-se o arroz e as carnes, representando $15,2 \%$ e 9,8\% da ingestão energética total, respectivamente. $O$ consumo de frutas representou $4,3 \%(n=492)$ e o de hortaliças $1,5 \%(n=768)$ da energia diária. Entre os ultraprocessados, embutidos, margarina e refrigerantes foram os produtos alimentícios mais consumidos, com contribuição energética de $5,6 \%(n=506), 3,4 \%(n=461)$ e $2,9 \%(n=323)$, respectivamente.

Os resultados da Tabela 2 mostram que o consumo de ultraprocessados foi significativamente maior entre as mulheres, $25,2 \%$ contra $22,9 \%$ entre homens $(\mathrm{p}=0,018)$, nos adultos sem cônjuge $(26,9 \% ; \mathrm{p}=0,001)$ e nos que possuíam plano de saúde $(26,2 \% ; \mathrm{p}=0,007)$. O consumo de ultraprocessados aumentou com a melhora dos níveis de renda $(p=0,003)$ e escolaridade $(p<0,001)$, e diminuiu com o incremento da idade, chegando a menos de $20,0 \%$ nos adultos de 50 a 59 anos $(\mathrm{p}<0,001)$ e com o aumento no número de filhos, passando de $23,1 \%$ em adultos com 1 a 2 filhos, para $19,2 \%$ com 3 ou mais filhos, em comparação com $28,8 \%$ para aqueles sem filhos.

Os resultados da Tabela 3 indicam que os indivíduos insuficientemente ativos (22,9\% $\mathrm{p}=0,004)$ ou inativos $(23,3 \% \mathrm{p}=0,004)$ no lazer tiveram menor consumo de ultraprocessados, quando comparados aos indivíduos ativos $(27,7 \%)$.

Os resultados do modelo de regressão linear múltipla estão apresentados na Tabela 4, e demonstram a maior participação energética dos ultraprocessados na alimentação de adultos do sexo feminino ( $\mathrm{p}=0,004)$, nos ex-fumantes $(\mathrm{p}=0,033)$, além de aumentar conforme o nível de escolaridade, chegando a 32,9\% no segmento com 12 anos ou mais de estudo, o mesmo ocorre conforme diminui a prática de atividade física, passando de $21,4 \%(\mathrm{p}=0,022)$ nos insuficientemente ativos para $22,5 \%(p=0,049)$ nos inativos. Em contraposição, contribuição energética dos ultraprocessados diminuiu com o avançar da idade, passando de $25,4 \%(\mathrm{p}<0,001)$ para $16,8 \%(\mathrm{p}<0,001)$ entre as faixas etárias de 20-29 e 50-59 anos.

\section{Discussão}

A análise da participação energética dos alimentos segundo a natureza, extensão e propósito do processamento aponta que o percentual de produtos alimentícios ultraprocessados na alimentação dos adultos $(24,1 \%)$ foi superior aos $21,5 \%$ encontrado por estudo que avaliou informações da população brasileira $(\geq 10 \text { anos })^{17}$ e está dentro do encontrado em estudo que avaliou domicílios brasileiros em geral, que apontou variação de $15,4 \%$ no menor quartil a $39,4 \%$ no maior ${ }^{7}$. Contudo, no Brasil, apesar da participação dos ultraprocessados no valor energético total estar aumentando, ainda é inferior à observada em países desenvolvidos como Canadá $(47,7 \%)^{11}$, Bélgica (44,6\%), Alemanha (46,2\%), Reino Unido $(50,7 \%)^{9}$ e México $(29,8 \%)^{10}$. Cabe ressaltar que ainda não foi estabelecido um limite máximo de participação no valor energético diário para os ultraprocessados, o que se recomenda é que o consumo seja o menor possível ${ }^{19}$.

$\mathrm{O}$ aumento do consumo de produtos ultraprocessados tem sido relacionado ao distanciamento da tradição culinária, que tem se tornado cada vez menos presente no dia a dia das pessoas e das famílias9. Isto pode ser explicado por mudanças no estilo de vida das famílias que foram impulsionadas pela participação da mulher no mercado de trabalho, pela urbanização e tecnologia, mas também pelo investimento da indústria alimentícia ao longo dos anos no desenvolvimento de características dos produtos ultraprocessados para torná-los hiperpalatáveis, prontos para o consumo, duráveis, práticos e disponíveis para todos os estratos sociais da população $0^{9,19}$. Dados da POF $2017-18^{20}$ revelam que as famílias com menores rendimentos destinam $22 \%$ de seus rendimentos para alimentação, enquanto entre as que têm maior rendimento ( $>25 \mathrm{SM}$ ) destinam $7,6 \%$, ou seja, quase três vezes menos.

Campinas é uma cidade de grande porte, tem mais de 1 milhão de habitantes, desde o censo de 2010, é conhecida como o Vale do Silício brasileiro graças à presença de mais de 30 unidades das maiores empresas high tech mundiais, além de ter 
Tabela 1. Médias de consumo diário e frequências relativas percentuais da contribuição energética de alimentos in natura ou minimamente processados, processados, ultraprocessados e ingredientes culinários na dieta de adultos (20 a 59 anos). ISACamp 2008-2009.

\begin{tabular}{|c|c|c|c|}
\hline Grupos de alimentos e itens de consumo & $\mathbf{n}$ & $\mathrm{Kcal} / \mathrm{dia}$ & $\begin{array}{l}\text { \% da ingestão } \\
\text { energética total }\end{array}$ \\
\hline In natura ou minimamente processados & 947 & 1068,3 & 54,1 \\
\hline Arroz & 814 & 291,6 & 15,2 \\
\hline Carnes (bovina, suína e vísceras) & 492 & 201,9 & 9,8 \\
\hline Outros cereais & 475 & 123,7 & 5,5 \\
\hline Feijão e outras leguminosas & 680 & 108,1 & 5,1 \\
\hline Carne de aves & 327 & 76,3 & 4,0 \\
\hline Frutas e sucos de frutas & 492 & 74,8 & 4,3 \\
\hline Leite & 574 & 74,4 & 4,0 \\
\hline Raízes e tubérculos & 300 & 40,7 & 2,0 \\
\hline Ovos & 401 & 27,4 & 1,4 \\
\hline Verduras e legumes & 768 & 25,7 & 1,5 \\
\hline Peixes & 59 & 14,0 & 0,8 \\
\hline Outros alimentos in natura ou minimamente processados ${ }^{\mathrm{a}}$ & 768 & 9,7 & 0,5 \\
\hline Processados & 773 & 211,6 & 10,6 \\
\hline Pão francês & 572 & 123,1 & 6,4 \\
\hline Queijos & 341 & 64,1 & 3,1 \\
\hline Carne processadas & 99 & 15,8 & 0,7 \\
\hline Enlatados & 242 & 8,6 & 0,4 \\
\hline Ultraprocessados & 907 & 502,1 & 24,1 \\
\hline Embutidos & 506 & 117,0 & 5,6 \\
\hline Margarina & 461 & 72,4 & 3,4 \\
\hline Refrigerantes & 323 & 63,6 & 2,9 \\
\hline Doces e sobremesas $^{\mathrm{b}}$ & 249 & 62,5 & 2,7 \\
\hline Biscoito salgado e salgadinho de pacote & 223 & 43,4 & 2,3 \\
\hline Pães de forma, hambúrguer e hot dog & 227 & 42,6 & 2,3 \\
\hline Outras bebidas açucaradas & 249 & 25,8 & 1,4 \\
\hline Gordura vegetal e animal $^{c}$ & 185 & 19,7 & 0,9 \\
\hline Outros ultraprocessados ${ }^{\mathrm{d}}$ & 638 & 55,1 & 2,6 \\
\hline Ingredientes culinários & 942 & 220,6 & 11,2 \\
\hline Óleos & 931 & 163,8 & 8,3 \\
\hline Açúcar & 692 & 56,8 & 2,9 \\
\hline Total & 949 & 2002,6 & 100,0 \\
\hline
\end{tabular}

${ }^{a}$ Café e chá sem adição de açúcares, especiarias, condimentos secos, oleaginosas sem sal/açúcar, chocolate em pó e água de coco; ${ }^{\mathrm{b} B i s c o i t o ~ d o c e, ~ s o r v e t e, ~ c h o c o l a t e, ~ b o l o s, ~ t o r t a s, ~ p u d i n s, ~ b a l a s ; ~}{ }^{\mathrm{C}}$ Gordura vegetal hidrogenada, requeijão e cream cheese; ${ }^{\mathrm{d}}$ Suplementos de vitaminas e minerais, essência de baunilha, fermento em pó, temperos prontos, substitutos de refeição e sopa em pó.

Fonte: Elaborado pelas autoras a partir dos dados do Inquérito ISACamp 2008-2009.

um IDH 0,805 contra 0,754 do país, ainda assim, apresenta uma taxa de desemprego semelhante à nacional de $12,34 \%$ contra $12,7 \%$, e mais de $30 \%$ da população apresenta um rendimento mensal menor que 0,5 salário mínimo ${ }^{21}$.

No Brasil, grande parte da população tem baixo poder aquisitivo, a ideia de praticidade associada ao baixo custo dos produtos ultraprocessados, e a influência das campanhas de marketing das multinacionais de alimentos tem gerado inúmeros prejuízos à cultura alimentar, à comensalidade e ao estado nutricional da população ${ }^{3,19,22}$.
Os resultados obtidos apontam que o arroz e o feijão contribuíram com $20,3 \%$ das calorias ingeridas, valor discretamente inferior ao encontrado para a população brasileira $(22,9 \%)$ por Louzada et al. ${ }^{17}$. Desde a década de 1970, dados de pesquisas de orçamentos familiares indicam quedas significativas na aquisição de alimentos básicos como arroz e o feijão ${ }^{8,23}$, entre os brasileiros. Segundo Silva ${ }^{24}$, o Brasil possui uma diversidade de culturas alimentares em suas macrorregiões e independentemente da classe social, o arroz com feiião constitui a base da alimentação. Contudo, 
Tabela 2. Médias de consumo diário e frequências relativas percentuais da contribuição energética de produtos alimentícios ultraprocessados na dieta de adultos (20 a 59 anos), segundo variáveis sociodemográficas. ISACamp 2008-2009.

\begin{tabular}{|c|c|c|c|}
\hline Variáveis e categorias & Kcal/dia & $\begin{array}{l}\text { \% da ingestão } \\
\text { energética total }\end{array}$ & Valor de $\mathrm{p}$ \\
\hline Total & 502,1 & 24,1 & \\
\hline \multicolumn{4}{|l|}{ Sexo } \\
\hline Masculino & 548,9 & 22,9 & \\
\hline Feminino & 639,8 & 25,2 & 0,018 \\
\hline \multicolumn{4}{|l|}{ Faixa etária (em anos) } \\
\hline $20-29$ & 674,4 & 29,2 & \\
\hline $30-39$ & 504,3 & 24,1 & $<0,001$ \\
\hline $40-49$ & 368,0 & 20,7 & $<0,001$ \\
\hline $50-59$ & 372,1 & 19,6 & $<0,001$ \\
\hline \multicolumn{4}{|l|}{ Escolaridade (em anos) } \\
\hline $0-7$ & 377,0 & 18,7 & \\
\hline $8-11$ & 521,6 & 24,5 & 0,001 \\
\hline $12 \mathrm{ou}+$ & 588,4 & 28,3 & $<0,001$ \\
\hline \multicolumn{4}{|c|}{ Renda familiar per capita (em salários mínimos) } \\
\hline$<1$ & 412,0 & 21,5 & \\
\hline$\geq 1 \mathrm{a} \leq 3$ & 559,4 & 25,2 & 0,011 \\
\hline$>3$ & 558,6 & 26,9 & 0,003 \\
\hline \multicolumn{4}{|l|}{ Situação conjugal } \\
\hline Com cônjuge & 463,2 & 22,4 & \\
\hline Sem cônjuge & 564,6 & 26,8 & 0,001 \\
\hline \multicolumn{4}{|l|}{ Número de filhos } \\
\hline Nenhum & 649,3 & 28,8 & \\
\hline $1-2$ & 470,0 & 23,1 & $<0,001$ \\
\hline $3 \mathrm{ou}+$ & 350,8 & 19,2 & $<0,001$ \\
\hline \multicolumn{4}{|l|}{ Posse de plano médico de saúde } \\
\hline Sim & 547,5 & 26,2 & \\
\hline Não & 466,5 & 22,4 & 0,007 \\
\hline
\end{tabular}

Fonte: Elaborado pelas autoras a partir dos dados do Inquérito ISACamp 2008-2009.

a globalização da economia, a industrialização e as novas demandas da vida urbana levaram à redução do consumo per capita dos alimentos mais tradicionais da cozinha brasileira ${ }^{25}$.

Os achados deste estudo revelam um consumo insuficiente de frutas e hortaliças, assim como verificado por outros autores ${ }^{26,27}$. Ao analisarem dados da Pesquisa Nacional de Saúde de 2013, Jaime et al. ${ }^{27}$ observaram que pouco mais de um terço da população $(37,3 \%)$ atingia o nível recomendado de ingestão de frutas e hortaliças, muito semelhante aos 34,6\% encontrados pelo Vigitel Brasil $2017^{28}$. Por outro lado, os dados mostram o predomínio dos alimentos in natura na alimentação (54,1\%), o que é desejável, ao mesmo tempo situam o refrigerante entre os produtos ultraprocessados mais consumidos. Segundo a POF 2008-2009, o refrigerante também aparece entre os itens de maior consumo diário per capita, chegando a 14,6\% a frequência de consumo em cinco ou mais dias da semana ${ }^{28}$ estando associado com o aumento de ingestão de energia e consequentemente das prevalências de obesidade e outras doenças crônicas ${ }^{26,29}$.

Considerando o cenário nacional de consumo insuficiente de frutas e hortaliças e de agravamento do perfil epidemiológico das doenças crônicas - $54 \%$ de sobrepeso, $18,9 \%$ de obesidade, $24,3 \%$ de HAS, 7,6\% de diabetes ${ }^{28}$-, e das evidências de associação destas com o consumo de ultraprocessados ${ }^{4}$, uma das metas do Plano de Ações Estratégicas para o Enfrentamento das Doenças Crônicas Não Transmissíveis no Brasil ${ }^{30}$ é aumentar o consumo de frutas e hortaliças até 2022, mediante o fortalecimento das políticas de alimentação escolar e regulação dos preços dos alimentos. Nesse sentido, o Ministério da Saúde assumiu o compromisso de ampliar em pelo menos $17,8 \%$ o 
Tabela 3. Médias de consumo diário e frequências relativas percentuais da contribuição energética de produtos alimentícios ultraprocessados na dieta de adultos (20 a 59 anos), segundo comportamentos relacionados à saúde e IMC. ISACamp 2008-2009.

\begin{tabular}{|c|c|c|c|}
\hline Variáveis e categorias & Kcal/dia & $\begin{array}{l}\text { \% da ingestão } \\
\text { energética total }\end{array}$ & Valor de $\mathrm{p}$ \\
\hline \multicolumn{4}{|l|}{ Prática de atividade física no lazer } \\
\hline Ativo & 627,2 & 27,7 & \\
\hline Insuficientemente ativo & 514,5 & 22,9 & 0,005 \\
\hline Inativo & 463,6 & 23,3 & 0,004 \\
\hline \multicolumn{4}{|l|}{$\operatorname{IMC}\left(\mathrm{kg} / \mathrm{m}^{2}\right)$} \\
\hline Baixo peso & 443,5 & 24,2 & 0,912 \\
\hline Eutrofia & 518,7 & 24,5 & \\
\hline Sobrepeso & 515,4 & 23,7 & 0,580 \\
\hline Obesidade & 431,6 & 22,7 & 0,218 \\
\hline \multicolumn{4}{|l|}{ Tabagismo } \\
\hline Nunca fumou & 523,8 & 24,5 & \\
\hline Fumante & 425,3 & 21,6 & 0,093 \\
\hline Ex-fumante & 509,7 & 26,2 & 0,366 \\
\hline \multicolumn{4}{|l|}{ Frequência de consumo de álcool } \\
\hline Não bebe & 486,4 & 23,7 & \\
\hline $1 \mathrm{vez} / \mathrm{semana}$ & 558,4 & 25,8 & 0,112 \\
\hline 2 vezes ou $+/$ semana & 412,4 & 20,8 & 0,167 \\
\hline
\end{tabular}

Tabela 4. Modelo de regressão linear múltiplo: variáveis associadas ao consumo de produtos alimentícios ultraprocessados em adultos. ISACamp 2008-2009.

\begin{tabular}{lrr}
\hline Variáveis e categorias & Média $($ IC95\%) & $\begin{array}{r}\text { Valor } \\
\text { de } \mathbf{~}\end{array}$ \\
\hline Sexo & & \\
Masculino & $25,4(21,3-29,6)$ & \\
Feminino & $28,3(22,3-34,3)$ & 0,004 \\
Faixa etária (em anos) & & \\
20-29 & $25,4(21,3-29,6)$ & \\
$30-39$ & $20,8(13,4-28,4)$ & 0,009 \\
$40-49$ & $17,7(10,6-24,9)$ & $<0,001$ \\
50-59 & $16,8(9,3-24,3)$ & $<0,001$ \\
Escolaridade (em anos) & & \\
0-7 & $25,4(21,3-29,6)$ & \\
$8-11$ & $29,2(21,7-36,7)$ & 0,029 \\
12 ou + & $32,9(25,3-40,6)$ & $<0,001$ \\
Tabagismo & & \\
Nunca fumou & $25,4(21,3-29,6)$ & \\
Fumante & $25,1(17,8-32,5)$ & 0,856 \\
Ex-fumante & $29,4(21,6-37,3)$ & 0,033 \\
Prática de atividade física & & \\
no lazer & & \\
Ativo & $25,4(21,3-29,6)$ & \\
Insuficientemente ativo & $21,4(13,9-29,0)$ & 0,022 \\
Inativo & $22,5(15,5-29,6)$ & 0,049 \\
\hline Fonte: Elaborado pelas autoras a partir dos dados do Inquérito \\
ISACamp 2008-2009. & & \\
& &
\end{tabular}

percentual de adultos que ingerem regularmente frutas e hortaliças até o ano de 2019.

O consumo de ultraprocessados mostrou-se maior no sexo feminino e entre os segmentos mais jovens. Resultado semelhante foi obtido por Bielemann et al. ${ }^{31}$ em estudo que encontrou um percentual de consumo maior entre as mulheres, além de apontar que mais da metade das 3.758 $\mathrm{kcal} /$ dia ingeridas por adultos jovens (idade média de 22,8 anos) eram provenientes de produtos ultraprocessados.

A redução do percentual de consumo de ultraprocessados conforme o aumento da idade dos adultos estudados, é um argumento em favor da cultura alimentar e das tradições culinárias como fundamentais para a preservação de hábitos alimentares mais saudáveis, na medida em que os adultos com 50 a 59 anos, no início dos anos 2000, só foram ter maior acesso à produtos ultraprocessados quando já eram jovens adultos. Contudo, esta hipótese precisa de mais investigações para confirmar o argumento.

A maior participação de ultraprocessados na alimentação das mulheres, de $1 / 4$ da média do consumo energético total $(25,2 \% \mathrm{p}=0,018)$ é preocupante, uma vez que pesquisas apontam que uma alimentação com maior conteúdo de ultraprocessados, que apresenta elevados teores de energia, gorduras, açúcares, sódio, além de 
baixo teor de fibras e micronutrientes, está relacionada ao aumento das prevalências de excesso de peso e outras doenças crônicas não transmissíveis $^{3,4,7,8,17,31}$. Ao analisarem a transição da saúde e da doença no Brasil, Souza et al. ${ }^{32}$ observam que as DCNT são a primeira causa de óbito no país $(75 \%)$ e que a alimentação inadequada representa um dos principais fatores de risco para doenças crônicas.

Ao analisar variáveis que são consideradas indicadores de vulnerabilidade social, observou-se que a escolaridade foi mantida no modelo, revelando um crescimento do consumo de alimentos ultraprocessados com o aumento dos anos de estudo. Martins et al. ${ }^{8}$ detectaram um gradiente de aumento da contribuição calórica dos ultraprocessados entre o primeiro $(16,6 \%)$ e o último quintil $(33,8 \%)$ da distribuição de renda da população brasileira. Resultado contrário foi verificado na França, mostrando menor consumo de ultraprocessados entre os adultos com níveis superiores de escolaridade e renda ${ }^{13}$. Estudo realizado no México também encontrou maior consumo de ultraprocessados nos estratos de maior renda e escolaridade do chefe de família ${ }^{10}$.

Vale ressaltar que mesmo não havendo ainda consenso na literatura sobre a associação entre ultraprocessados e nível socioeconômico, houve aumento no consumo destes produtos em todos os estratos sociais, e que os mercados mais promissores para a indústria de alimentos não são mais os países ricos da América do Norte e da Europa, e sim os países em desenvolvimento e com baixa renda. No que se refere ao volume de vendas de produtos ultraprocessados, apesar de ainda ser maior em países de alta renda, a taxa de crescimento nos países pobres foi de $48,0 \%$ enquanto na América do Norte foi de 2,3\%, entre os anos de 2000 e $2013^{3}$.

Não há controvérsia sobre o avanço que representa a conquista da inclusão do direito à alimentação adequada e saudável entre os direitos sociais básicos da população brasileira, no entanto deve-se considerar a grave desigualdade social que atinge a sociedade determinando distintos prejuízos ao padrão alimentar. A incorporação de novos hábitos alimentares pelos estratos mais pobres da população tem sido impulsionada pela publicidade de alimentos e pelo barateamento dos produtos ultraprocessados ${ }^{19,33}$. Monteiro e Castro $^{33}$ citam algumas estratégias utilizadas pelas indústrias de alimentos para induzir a compra de ultraprocessados entre os consumidores de baixa renda como, por exemplo, o desenvolvimento de produtos fortificados, de embalagens econômicas e da criação de novos canais de co- mercialização, de maneira a facilitar o acesso, independentemente do estrato social:

Uma coisa é comer biscoito e beber refrigerante assistindo televisão ao retornar da escola, como fazem os filhos da classe de maior renda, outra coisa é um garoto pobre comer biscoito e beber refrigerante porque é um modo barato de saciar a fome $e^{34}$.

Nesse sentido, a Segurança Alimentar e Nutricional e, portanto, o Direito Humano à Alimentação Adequada e Saudável da população brasileira segue sob ameaça, não só pelo modelo de desenvolvimento econômico em detrimento do desenvolvimento social vigente no país, como também pelas estratégias de ampliação de mercado das grandes corporações.

Os resultados deste estudo apontam que os ex-fumantes apresentaram maior consumo de produtos alimentícios ultraprocessados $(29,4 \%)$, o que contrasta com os resultados obtidos por Tian et al. ${ }^{35}$ em pesquisa que verificou que exfumantes australianos adotam comportamentos alimentares e práticas de atividade física mais saudáveis. É relatada na literatura uma relação entre a cessação do tabagismo e o maior ganho de peso, o que poderia indicar uma substituição de um comportamento inadequado por outro. Há hipóteses de que o uso de substâncias psicoativas e o consumo alimentar atingem áreas cerebrais comuns, destacando-se os alimentos ricos em açúcares e gorduras ${ }^{36}$.

Sobre os hábitos de vida, observou-se que a contribuição energética dos produtos ultraprocessados foi maior nos indivíduos fisicamente ativos no lazer. Uma hipótese que poderia explicar este resultado, bem como o maior consumo no sexo feminino e entre os mais jovens é a infinidade de produtos ultraprocessados disponíveis no mercado com apelo fitness. O culto ao corpo magro que existe na sociedade contemporânea gera oportunidades para as indústrias de alimentos investirem no desenvolvimento de versões light, diet, low carb ou fitness dos produtos, que induzem os consumidores a acreditarem que são mais saudáveis ${ }^{33}$. Os achados sugerem que a maior participação dos ultraprocessados na alimentação dos indivíduos fisicamente ativos no lazer, bem como nos segmentos de maior escolaridade e nas mulheres pode decorrer da falta de conhecimento a respeito dos prejuízos que esses produtos causam para a saúde. Contudo, são hipóteses que necessitam ainda de investigação.

Dentre as limitações do estudo destaca-se o desenho transversal, que impossibilita a verificação de relações causais, mesmo este não sendo o objetivo proposto. A aplicação de um único recordatório de 24 horas, poderia ser também um 
limitador por não permitir estimar a ingestão usual dos indivíduos, devido à variabilidade do consumo alimentar. A ausência de associação entre o consumo de ultraprocessados e o IMC pode decorrer do sub-relato da ingestão alimentar nos adultos com excesso de peso ${ }^{37}$. Entretanto, por tratar-se de um estudo de base populacional, em que o método de coleta dos R24h garantiu que fossem obtidos dados de diferentes dias da semana e diferentes meses do ano, como forma de contemplar a variabilidade, foi possível estimar uma média de ingestão para a população avaliada. Por outro lado, o estudo alcançou seu objetivo e pode contribuir com a construção de evidências sobre o consumo de alimentos ultraprocessados em adultos.

Cabe ressaltar o fato de serem resultados de um inquérito de saúde de base populacional periódico, em uma cidade brasileira de grande porte, o que permite o desenvolvimento de estudos de monitoramento que verifiquem mudanças no padrão de consumo de ultraprocessados ao longo dos anos.

\section{Colaboradores}

MG Pereira fez a programação das análises estatísticas e participou da análise dos resultados e redação do manuscrito. D Assumpção produziu o banco de dados com a classificação de grau de processamento de alimentos, participou da programação das análises estatísticas, da análise dos resultados e da redação do manuscrito. MBA Barros coordenou o Inquérito de Saúde ISACamp 2008-2009, revisou as análises estatísticas e fez a leitura crítica do manuscrito. LTO Zangirolani participou da construção do plano de análises estatísticas, da análise dos resultados, da redação do manuscrito e da leitura crítica do mesmo. Todos os autores aprovaram a versão final do manuscrito.
Mesmo reconhecendo que o Brasil avançou muito em políticas públicas e ações voltadas ao enfrentamento de questões relativas à alimentação e nutrição nos últimos 15 anos, e que a implantação e capilaridade das políticas e ações não são tarefas triviais, o aumento da prevalência da obesidade e de outras DCNT no Brasil, segue avançando, evidenciando não só as contradições do modelo de desenvolvimento do país, mas também que ainda há tempo de intervir positivamente em favor da saúde alimentar da população brasileira.

Avaliar os resultados dessas políticas e ações para subsidiar os aprimoramentos e o enfrentamento ao desmonte das políticas públicas no país, bem como fortalecer a discussão em torno da natureza, extensão e propósito do processamento de alimentos são prioridades na conjuntura atual. Nessa perspectiva, identificar os comportamentos e os fatores associados ao consumo dos ultraprocessados é indispensável, pois além dos prejuízos trazidos à qualidade da alimentação e consequentemente à saúde, há também fatores econômicos, sociais e ambientais envolvidos.

\section{Referências}

1. Monteiro CA. Nutrition and health. The issue is not food, nor nutrients, so much as processing. Public Health Nutr 2009; 12(5):729-731.

2. Pollan M. Dos alimentos aos nutrientes. In: Pollan M. Em defesa da comida: um manifesto. Rio de Janeiro: Intrínseca; 2008. p.25-33.

3. Organização Pan-Americana da Saúde (OPAS). Alimentos e bebidas ultraprocessados na América Latina: tendências, efeito na obesidade e implicações para políticas públicas. Brasília: OPAS; 2018.

4. Monteiro CA, Cannon G, Lawrence M, Louzada MLC, Machado PP. Ultra-processed foods, diet quality, and health using the NOVA classification system. Rome: FAO; 2019

5. Rauber F, Louzada MLC, Steele EM, Millett C, Monteiro CA, Levy RB. Ultra-Processed Food Consumption and Chronic Non-Communicable Diseases-Related Dietary Nutrient Profile in the UK (2008-2014). Nutrients 2018; 10:587.

6. Monteiro CA, Cannon G, Moubarac J-C, Levy RB, Louzada MLC, Jaime PC. The UN Decade of $\mathrm{Nu}-$ trition, the NOVA food classification and the trouble with ultra-processing. Public Health Nutr 2017; 21(1):5-17.

7. Canella DS, Levy RB, Martins AP, Claro RM, Moubarac J-C, Baraldi LG, Cannon G, Monteiro CA. Ultra-processed food products and obesity in Brazilian households (2008-2009). PLoS One 2014; 9(3):e92752. 
8. Martins APB, Levy RB, Claro RM, Moubarac JC, Monteiro CA. Participação crescente de produtos ultraprocessados na dieta brasileira (1987-2009). Rev Saude Publica 2013; 47(4):656-665.

9. Monteiro CA, Moubarac JC, Levy RB, Canella DS, Louzada MLC, Cannon G. Household availability of ultra-processed foods and obesity in nineteen European countries. Public Health Nutr 2017; 21(1):18-26.

10. Marrón-Ponce JA, Sánchez-Pimienta TG, Louzada MLC, Batis C. Energy contribution of NOVA food groups and sociodemographic determinants of ultra -processed food consumption in the Mexican population. Public Health Nutr 2018; 21(1):87-93.

11. Moubarac J-C, Batal M, Louzada ML, Martines Steele E, Monteiro, CA. Consumption of ultra-processed foods predicts diet quality in Canada. Appetite 2017; 108:512-520.

12. Julia $C$, Martinez L, Allès $B$, Touvier $M$, Hercberg $S$, Méjean C, Kesse-Guyot E. Contribution of ultra-processed foods in the diet of adults from the French NutriNet-Santé study. Public Health Nutr 2017; 21(1):2737.

13. Schnabel L, Kesse-Guyot E, Allès B, Touvier M, Srour B, Hercberg S, Buscail C, Julia C. Association Between Ultraprocessed Food Consumption and Risk of Mortality Among Middle-aged Adults in France. JAMA Intern Med 2019; 179(4):490-498.

14. Thompson FE, Byers T. Dietary assessment resource manual. J Nutr 1994; 124(Supl.):2245-2317.

15. Fisberg RM, Villar BS. Manual de receitas e medidas caseiras para cálculo de inquéritos alimentares. São Paulo: Editora Signus; 2002.

16. Pinheiro ABV, Lacerda EMA, Benzecry EH, Gomes MCS, Costa VM. Tabela para avaliação de consumo alimentar em medidas caseiras. São Paulo: Editora Atheneu; 2004.

17. Louzada MLC, Martins APB, Canella DS, Baraldi LG, Levy RB, Claro RM, Moubarac J-C, Cannon G, Monteiro CA. Alimentos ultraprocessados e perfil nutricional da dieta no Brasil. Rev Saude Publica 2015; 49:38.

18. World Health Organization (WHO). Physical status: the use and interpretation of anthropometry. Genebra: WHO; 1995.

19. Brasil. Ministério da Saúde (MS). Secretaria de Atenção à Saúde. Departamento de Atenção Básica. Guia Alimentar para a População Brasileira. Brasília: MS; 2014.

20. Instituto Brasileiro de Geografia e Estatística (IBGE). Pesquisa de Orçamentos Familiares 2017-2018: primeiros resultados. Rio de Janeiro: IBGE; 2019.

21. Instituto Brasileiro de Geografia e Estatística (IBGE). Campinas. Panorama [Internet]. [acessado 2019 out 11]. Disponível em: https://cidades.ibge.gov.br/brasil/ sp/campinas/panorama.

22. Monteiro CA, Cannon G, Levy RB, Moubarac J-C, Jaime PC, Martins AP, Canella D, Louzada M, Parra D, Ricardo C, Calixto G, Machado P, Martins C, Martinez E, Baraldi L, Garzillo J, Sattamini I. NOVA. A estrela brilha. World Nutr 2016; 7(1-3):28-40.

23. Levy-Costa RB, Sichieri R, Pontes NS, Monteiro CA. Disponibilidade domiciliar de alimentos no Brasil: distribuição e evolução (1974-2003). Rev Saude Publica 2005; 39(4):530-540.

24. Silva SMCS. Padrões brasileiros: respeito à diversidade culinária. In: Miranda DS, Cornelli G. Cultura e alimentação: saberes alimentares e sabores culturais. São Paulo: SESC-SP; 2007.
25. Diez Garcia RW. Reflexos da globalização na cultura alimentar: considerações sobre as mudanças na alimentação urbana. Rev Nutr 2003; 16(4):483-492.

26. Souza AM, Pereira RA, Yokoo EM, Levy RB, Sichieri R. Alimentos mais consumidos no Brasil: Inquérito Nacional de Alimentação 2008-2009. Rev Saude Publica 2013; 47(1 Supl.):190S-199S.

27. Jaime PC, Stopa SR, Oliveira TP, Vieira ML, Szwarcwald CL, Malta DC. Prevalência e distribuição sociodemográfica de marcadores de alimentação saudável, Pesquisa Nacional de Saúde, Brasil 2013. Epidemiol Serv Saude 2015; 24(2):267-276.

28. Brasil. Ministério da Saúde (MS). Secretaria de Vigilância em Saúde. Departamento de Vigilância de Doenças e Agravos não Transmissíveis e Promoção de Saúde. Vigitel Brasil 2017: vigilância de fatores de risco e proteção para doenças crônicas por inquérito telefônico: estimativas sobre frequência e distribuição sociodemográfica de fatores de risco e proteção para doenças crônicas nas capitais dos 26 estados brasileiros e no Distrito Federal em 2017. Brasília: MS, 2018.

29. Vartanian LR, Schwartz MB, Brownell KD. Effects of Soft Drink Consumption on Nutrition and Health: A Systematic Review and Meta-Analysis. Am J Public Health 2007; 97(4):667-675.

30. Brasil. Ministério da Saúde (MS). Secretaria de Vigilância em Saúde. Departamento de Análise de Situação de Saúde. Plano de ações estratégicas para o enfrentamento das Doenças Crônicas Não Transmissíveis (DCNT) no Brasil 2011-2022. Brasília: MS; 2011.

31. Bielemann RM, Motta JVS, Minten GC, Horta BL, Gigante DP. Consumo de alimentos ultraprocessados e impacto na dieta de adultos jovens. Rev Saude Publica 2015; 49:28.

32. Souza MFM, Malta DC, França EB, Barreto ML. Transição da saúde e da doença no Brasil e nas Unidades Federadas durante os 30 anos do Sistema Único de Saúde. Cien Saude Colet 2018; 23(6):1737-1750.

33. Monteiro CA, Castro IRR. Por que é necessário regulamentar a publicidade de alimentos. Cien Cult 2009; 61(4):56-59.

34. Maluf RS. Segurança alimentar e nutricional com valorização da cultura alimentar. In: Miranda DS, Cornelli G, organizadores. Cultura e alimentação - saberes alimentares e sabores culturais. São Paulo: SESC; 2007. p. 143-150.

35. Tian J, Gall SL, Smith KJ, Dwyer T, Venn AJ. Worsening Dietary and Physical Activity Behaviors Do Not Readily Explain Why Smokers Gain Weight After Cessation: A Cohort Study in Young Adults. Nicotine Tob Res 2017; 19(3):357-366.

36. Ferreira IB, Paiva CB, Narvaez JCM, Bosa VL. Estado nutricional e hábitos alimentares de dependentes químicos em tratamento ambulatorial. J Bras Psiquiatr 2015; 64(2):146-153.

37. Avelino GF, Previdelli AN, Castro MA, Marchioni DML, Fisberg RM. Sub-relato da ingestão energética e fatores associados em estudo de base populacional. Cad Saude Publica 2014; 30(3):663-668.

Artigo apresentado em 23/04/2019

Aprovado em 20/12/2019

Versão final apresentada em 22/12/2019

Editores-chefes: Romeu Gomes, Antônio Augusto Moura da Silva 